\begin{tabular}{|c|l|}
\hline Title & STM studies on electronic charge order in the PG state of Bi2212 \\
\hline Author(s) & Liu, Y.H.; Takeyama, K.; Ishikura, M.; Momono, N.; Oda, M.; Ido, M. \\
\hline Citation & $\begin{array}{l}\text { Physica C : Superconductivity, 460-462(2), 961-962 } \\
\text { https://doi.org/40.1016/.physc.2007.03.327 }\end{array}$ \\
\hline Issue Date & 2007-09-01 \\
\hline Doc URL & http://hdl.handle.net/2115/30198 \\
\hline Type & article (author version) \\
\hline File Information & PHYC460-462.pdf \\
\hline
\end{tabular}

Instructions for use 


\title{
STM Studies on Electronic Charge Order in the PG State of Bi2212
}

\author{
Y. H. Liu, K. Takeyama, M. Ishikura, N. Momono, M. Oda and M. Ido \\ Department of Physics, Hokkaido University, Sapporo 060-0810, Japan
}

\begin{abstract}
Using scanning tunneling microscopy (STM), we confirmed a nondispersive $\sim 4 a_{0} \times 4 a_{0}$ electronic charge order, whose characteristic energy is the pseudogap (PG), in the PG state of Bi2212 above $T_{\mathrm{c}}$.
\end{abstract}

Key words: STM/STS; $\mathrm{Bi}_{2} \mathrm{Sr}_{2} \mathrm{CaCu}_{2} \mathrm{O}_{8}$; pseudogap; electronic charge order PACS: 68.37.Ef, 74.72.Hs, 74.50.+r

\section{Introduction}

In high- $T_{\mathrm{c}}$ cuprates, the origin of PG and its relation to the superconductivity remain under debate. It has been widely thought that a hidden order dominates the PG state above $T_{\mathrm{c}}$. Recently, in the PG state of $\mathrm{Bi}_{2} \mathrm{Sr}_{2} \mathrm{CaCu}_{2} \mathrm{O}_{8+\delta}(\mathrm{Bi} 2212)$, Vershinin et al. [1] found a charge order in a two-dimensional (2-d) map of energy-resolved STS conductance, which is proportional to the local density of states (LDOS) at a quasiparticle energy. The charge order is oriented along the $\mathrm{Cu}-\mathrm{O}$ directions; its period, $\sim 5 a_{0}\left(a_{0}\right.$ : lattice constant), is independent of energy, which is called "nondispersive". Interestingly, it becomes evident in LDOS maps at low energies within the PG, suggesting that the nondispersive charge order may be a hidden order in the PG state above $T_{\mathrm{c}}$.

However, no other groups reported such a charge order above $T_{\mathrm{c}}$. In this study, therefore, we performed STM experiments above $T_{\mathrm{c}}$ on slightly UD Bi2212 to confirm the nondispersive charge order in the PG state. STM imaging provides a 2-d map reflecting the integration of LDOS from the Fermi level $E_{\mathrm{F}}$ to $E_{\mathrm{F}}+e V_{\mathrm{s}}\left(V_{\mathrm{s}}\right.$ : bias voltage), and can also

\footnotetext{
Email address: liu@topology.coe.hokudai.ac.jp (Y. H. Liu).
}

Preprint submitted to Elsevier Science be expected to detect the nondispersive charge order clearly because its period is energy-independent.

\section{Experimental}

Single crystals of Bi2212 were grown by TSFZ method. The hole doping level $p$ and $T_{\mathrm{c}}$ are $\sim 0.14$ and $81 \mathrm{~K}$, respectively. To obtain clean surfaces which are indispensable for STM experiments, the samples were cleaved in ultrahigh vacuum better than $10^{-9}$ torr before being inserted in situ into an STM head whose temperature is $5 \mathrm{~K}$. STM experiments in the $\mathrm{PG}$ state were performed after finishing those at $T \ll T_{\mathrm{c}}$ and warming the sample gradually up to a temperature, $85 \mathrm{~K}$, above $T_{c}$.

\section{Results and discussion}

Figure 1 (a) shows an STM image, measured in the constant-height mode at a bias voltage of $V_{\mathrm{s}}=$ $40 \mathrm{mV}$ and $T=85 \mathrm{~K}$. As has already been discussed in Refs. [2] and [3], in STM experiments on Bi2212 cleaved surfaces, where the top atomic plane closest to the STM tip is the semiconducting Bi-O plane with an energy gap $E_{\mathrm{g}}$ of the order of $100 \mathrm{meV}$, the second the insulating Sr-O plane and the third the 

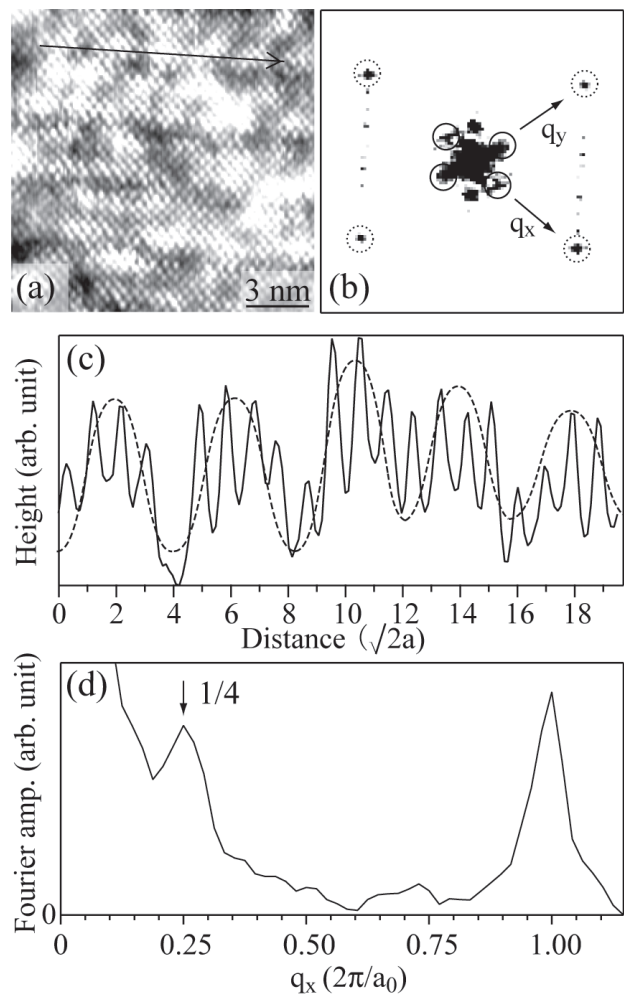

Fig. 1. (a) STM image in the PG state of Bi2212, measured at $V_{\mathrm{s}}=40 \mathrm{mV}$ and $85 \mathrm{~K}$. (b) Fourier map of the image. (c) Line profile taken along the solid line with an arrow on the image. The dashed line is a guide to the eye. (d) Line cut taken along the $q_{\mathrm{x}}$ direction on the Fourier map.

metallic or superconducting ( $\mathrm{SC}$ ) $\mathrm{Cu}-\mathrm{O}$ plane, we can image the $\mathrm{Cu}-\mathrm{O}$ plane at $V_{\mathrm{s}}<E_{\mathrm{g}} / e \sim 100 \mathrm{mV}$, because the $\mathrm{Bi}-\mathrm{O}$ and $\mathrm{Sr}-\mathrm{O}$ planes have no electronic states in the energy range from $E_{\mathrm{F}}$ to $E_{\mathrm{F}}+e V_{\mathrm{s}}$, contributing to tunneling. Indeed, a 1-d superstructure with periodic missing atom rows, which is a characteristic feature of the $\mathrm{Bi}-\mathrm{O}$ plane, is rather weak in the STM image observed at $V_{\mathrm{s}}=40 \mathrm{mV}$, suggesting that the low-bias image reflects the $\mathrm{Cu}-\mathrm{O}$ plane dominantly. Interestingly, a 2-d charge order can be seen clearly, especially for an upper part of the STM image, in addition to distinguished bright dots, corresponding to $\mathrm{Cu}$-sites. The 2 - $\mathrm{d}$ charge order is oriented along the $\mathrm{Cu}-\mathrm{O}$ directions, tilted by $45^{\circ}$ from the very weak 1-d superstructure.

Shown in Fig. 1 (c) is the line profile along a trace at $45^{\circ}$ from the $\mathrm{Cu}-\mathrm{O}$ direction or the orientation of the 2-d charge order, indicated by the solid line with an arrow in the STM image (Fig. 1 (a)). In this line profile, the period can be estimated to be about $4 a_{0}$ along the $\mathrm{Cu}-\mathrm{O}$ directions, where $a_{0}$ is the lattice constant or the nearest neighbor $\mathrm{Cu}-\mathrm{Cu}$ distance. The Fourier transform of the STM image is also useful to determine the period of the 2-d charge order. The Fourier map is shown in Fig. 1 (b), where four spots surrounded by dotted circles correspond to the Bragg reflections and four broad spots surrounded by solid circles near the origin are due to the periodic charge order. The intense peak in the line cut (Fig. 1 (d)) taken along the $q_{x}$ direction on the Fourier map (one of the $\mathrm{Cu}-\mathrm{O}$ directions in the real space) appears at $\sim 2 \pi / 4 a_{0}$, indicating that the period along the $\mathrm{Cu}-\mathrm{O}$ direction is $\sim 4 a_{0}$. A similar intense spot is also observed at $\sim 2 \pi / 4 a_{0}$ along the $q_{y}$ direction in the Fourier map (the other $\mathrm{Cu}-\mathrm{O}$ direction in the real space). These results indicate that the period of the 2-d charge order in the PG state above $T_{\mathrm{c}}$ is $\sim 4 a_{0} \times 4 a_{0}$. Furthermore, it has been found from the $V_{\mathrm{s}}$ dependence of Fourier spots corresponding to the charge order that the period is independent of energy and the intensity becomes evident at low energies within the PG. Thus, we have confirmed the nondispersive $\sim 4 a_{0} \times 4 a_{0}$ charge order whose characteristic energy is the PG above $T_{\mathrm{c}}$, although the period obtained in the present STM experiments is slightly smaller than that reported by Vershinin et al. [1].

However, we obtained the result that there also exist many Bi2212 samples in which the charge order is unclear even though they exhibit similar PG behavior above $T_{\mathrm{c}}$. Recently, a similar charge order, whose amplitude is strongly sample-dependent, has been found in the SC state [3-5]. More interestingly, in samples which exhibit strong $\sim 4 a_{0} \times 4 a_{0}$ charge orders at $T \ll T_{\mathrm{c}}$, the spatial dependence of energy gap structure is very inhomogeneous in nanometer scale, and vice vasa. Whether such a correlation between the electronic charge order and the gap inhomogeneity also holds in the PG state will be of great interest for the understanding of its origin.

Acknowledgements: This work was partly supported by Grant-in-Aids for Scientific Research and the 21st century COE program "Topological Science and Technology" from the Ministry of Education, Culture, Sports, Science and Technology of Japan.

\section{References}

[1] M. Vershinin, et al., Science 33 (2004) 1995.

[2] M. Oda, et al., Phys. Rev. B 53 (1996) 2253.

[3] A. Hashimoto, et al., cond-mat/0512496.

[4] N. Momono, et al., J. Phys. Soc. Jpn. 74 (2005) 2400.

[5] C. Howald, et al., Phys.Rev. B 67 (2003) 014533. 